\title{
Spectral and Light Variability of Be Star 60 Cygni
}

\author{
P. Koubský ${ }^{1}$, P. Harmanec ${ }^{1}$, A.M. Hubert ${ }^{2}$, M. Floquet ${ }^{2}$, J. Kubát ${ }^{1}$, \\ D. Ballereau ${ }^{2}$, H. Božic ${ }^{3}$, J. Chauville $^{2}$, D. Holmgren ${ }^{4}$, S. Yang ${ }^{5}$, \\ H. Cao ${ }^{6}$, P. Eenens ${ }^{7}$, L. Huang ${ }^{6}$, J.R. Percy ${ }^{8}$ \\ ${ }^{1}$ Astronomický ústav AV ČR, CZ-251 65 Ondřejov,Czech Republic \\ ${ }^{2}$ Observatoire de Paris-Meudon, 92195 Meudon, France \\ ${ }^{3}$ Hvar Observatory, Zagreb University, 10000 Zagreb, Croatia \\ ${ }^{4}$ Brandon University, Brandon, MA, R7A 6A9, Canada \\ ${ }^{5}$ Department of Physics and Astronomy, University of Victoria, PO Box \\ 3055 STN CSC, Victoria, B.C., V8W 3P6, Canada \\ ${ }^{6}$ Beijing Astronomical Observatory, Chinese Academy of Sciences, \\ Beijing 100080, China \\ ${ }^{7}$ Universidad de Guanajuato, 36000 Guanajuato, GTO, Mexico \\ ${ }^{8}$ College and Department of Astronomy, University of Toronto, \\ Mississauga, ON L5L IC6, Canada
}

\begin{abstract}
The analysis of high S/N spectra and photometric data of 60 Cyg shows that: (1) Pronounced long-term variations in spectra are accompanied with light variations. (2) The medium-term variations of RV coild indicate that $60 \mathrm{Cyg}$ is a spectroscopic binary. (3) Two independent structures dominate the rapid variability of both spectrum and light of the star.
\end{abstract}

\section{Introduction}

60 Cyg (V1931 Cyg, HD 200310, HR 8053, MWC 360; B1 Ve, $V=5$ m37 (var.), $v \sin i=320 \mathrm{~km} \mathrm{~s}^{-1}$ ), has been known as an emission-line star for a long time. Variable radial velocity was announced by Plaskett \& Pearce (1931), who reported it as a spectroscopic binary with a velocity range of $57 \mathrm{~km} \mathrm{~s}^{-1}$ but without any information about the period. Harmanec et al. (1986) analysed their and other published radial velocities together with available $U B V$ photometry and reported the presence of a period of 2.48 in both data sets. Percy et al. $(1988,1997)$ confirmed the presence of rapid light variations of 60 Cyg on a time scale of days. However, they were unable to confirm the 2.48 period. Recently, Hubert \& Floquet (1998) analysed the Hipparcos photometry of 60 Cyg and also concluded that the star is a rapid light variable, with a possible period of 0.3 .

The available records of spectroscopic observations of 60 Cyg (Copeland \& Heard 1963, Andrillat \& Houziaux 1967, Hubert-Delplace \& Hubert 1979, Fontaine et al. 1982, Slettebak 1982, Andrillat 1983 and Andrillat \& Houziaux 1991) clearly indicate the presence of pronounced long-term variations of the 

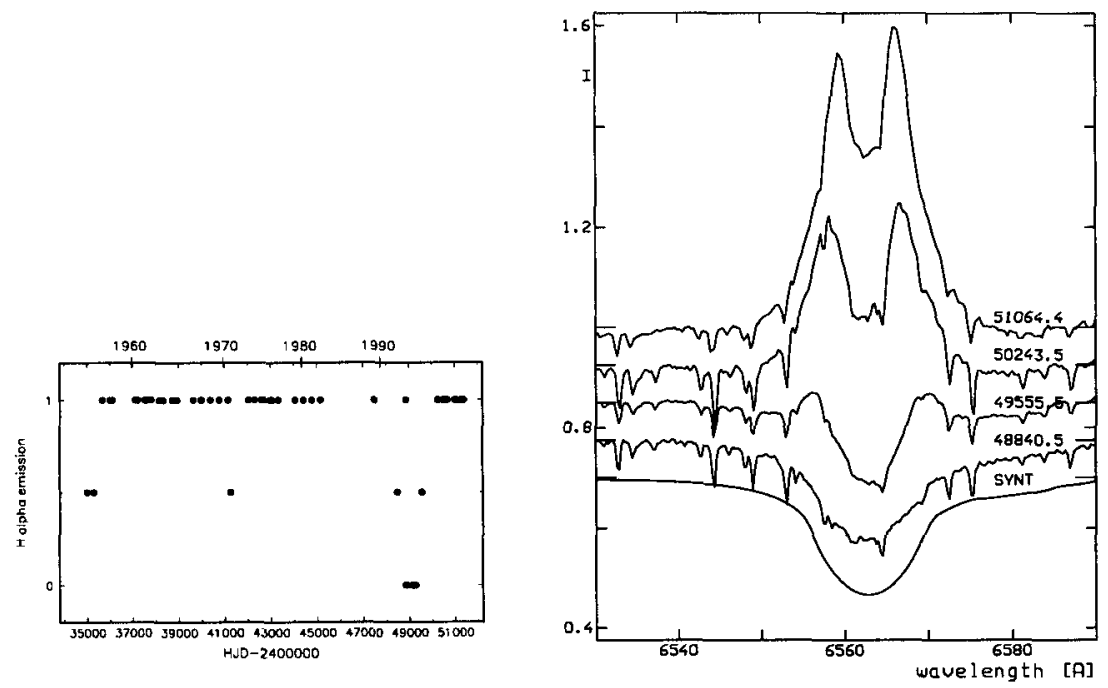

Figure 1. a) - left panel: Schematic representation of $\mathrm{H} \alpha$ versus time. 1 - emission, 0 - absorption; b) - right panel: Different types of $\mathrm{H} \alpha$ profile of $60 \mathrm{Cyg}$ identified by HJD-2400000 (synthetic spectrum for $T_{\text {eff }}=27000 \mathrm{~K}, \log g=4, v \sin i=320 \mathrm{~km} \mathrm{~s}^{-1}$ ).

Balmer emission lines. Rapid line-profile variations of the He I $4471 \AA$ line of 60 Cyg were discovered by Koubský et al. (1995).

60 Cyg was included among the targets of an international photometric campaign on bright $\mathrm{Be}$ stars during which it was rather intensively observed at Hvar (Harmanec et al. 1986, Pavlovski et al. 1997, Harmanec et al. 1997), Toronto (Percy et al. 1988, 1997) and Xinglong Observatories. To get a better insight into the character of rapid light variations observed, new series of $U B V$ observations were secured from San Pedro Mártir and Hvar during summer 1998 and 1999. It was also put on spectroscopic observing programs at the Haute Provence, Ondřejov, and Dominion Astrophysical Observatories.

In this poster we bring new results on long, medium and short term variability of 60 Cygni. Detailed version of the study will be published in Astronomy and Astrophysics.

\section{Long-term variations}

The existing records seem to indicate that the maximum strength of the $\mathrm{H} \alpha$ emission never exceeded a peak intensity of about 2.0 of the continuum level. The star underwent two long-lasting $\mathrm{H} \alpha$ emission episodes between 1953 and 1990, each of them some ten years long (see Fig. 1a). The intervals when no emission in $\mathrm{H} \alpha$ was detected were relatively short. The profile then mimicked a "normal" B type spectrum. In the phase of an intermediate strength of the $\mathrm{H} \alpha$ emission a relatively narrow absorption is flanked by faint emission components. We show the three types of $\mathrm{H} \alpha$ profile in Fig. 1b, together with the synthetic 

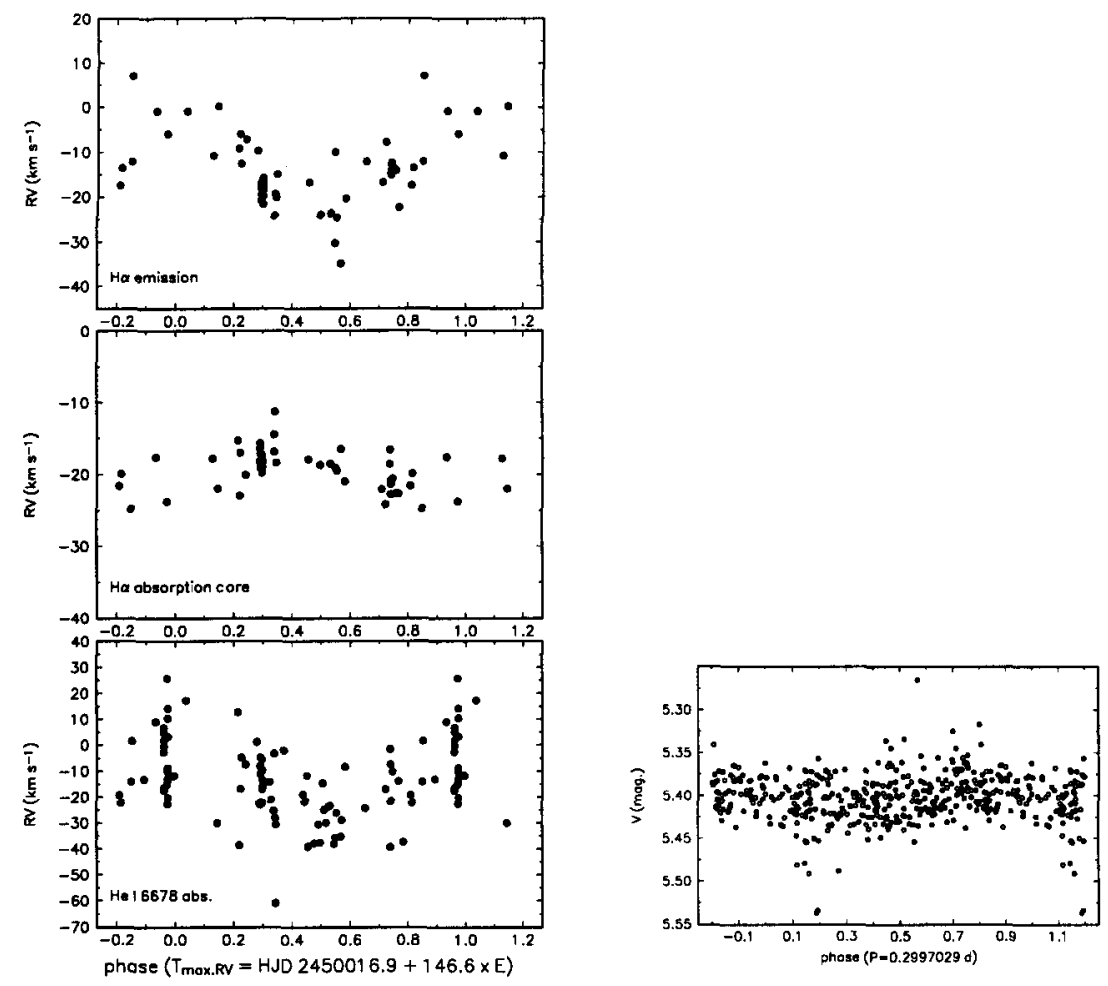

Figure 2. a) - left panel: Periodic RV variations of $\mathrm{H} \alpha$ and $\mathrm{He}$ I $6678 \AA \AA$ lines folded with the 147-day period; b) - right panel: Individual $\mathrm{V}$ mag. of $60 \mathrm{Cyg}$ folded with the period of $0.2997029 \mathrm{~d}$.

spectrum. The existing spectroscopic and photometric observations allow to track the correlation between the brightness of the star and the evolution of its envelope. Secular brightening was accompanied by the development of the Balmer emission in the spectra of 60 Cygni. This behaviour was classified by Harmanec $(1983,1994)$ as a positive correlation which is observed in objects where the disc (envelope) is seen under an intermediate angle.

\section{Medium-term variations}

The spectrum of 60 Cygni may vary also on the time scale of hundreds days or shorter. In some cases the medium-term spectral changes of Be stars were found to be related to the binary nature of the stars in question. We therefore tried to measure the spectra of $60 \mathrm{Cygni}$ for the radial velocities. In the case of $60 \mathrm{Cyg}$ this is not an easy task as all profiles of helium lines are strongly affected both by rapid and long term variability. To overcome the problem we measured the wings of the $\mathrm{H} \alpha$ emission and also the central absorption core. Using Stellingwerf's (1978) PDM period search techniques, we carried out a period search in several 
observed quantities: emission and absorption RV of $\mathrm{H} \alpha$, mean bisector RV of He I $6678 \AA$. The search was performed over the range of periods from $0 d_{2} 2$ to $200 \mathrm{~d} 0$. The best fit was found in all cases for periods near 150 days. It seems that the most probable period is close to 146.6 . Corresponding phase diagrams are presented in Fig. 2a. However, the 147-day period was not detected in the photometric data even in the case when the long-term trend was removed.

\section{Rapid variations}

The analysis of rapid variations shows that there are two independent patterns of variations on the short-term scale. The variations of helium profiles of $60 \mathrm{Cygni}$ obtained in nightly series were analysed with three different methods. (CLEAN, least-squares sinusoid fit, PDM analysis of local RV measured at specific levels). These analysis indicated a single frequency of $1.88 \mathrm{~cd}^{-1}$ or its alias $0.39 \mathrm{~cd}^{-1}$. The acceleration curves of the moving bumps can be folded with a period 0.2997 which is clearly seen in all photometric data. The phase diagram is presented in Fig. 2b.

\section{References}

Andrillat, Y. 1983, A\&AS 53, 319

Andrillat, Y., Houziaux, L. 1967, J. Observateurs 50, 107

Andrillat, Y., Houziaux, L. 1991, Inf. Bull. Var. Stars No. 3684

Copeland, J.A., Heard, J.F. 1963, Publ. David Dunlap Obs. 2, 317

Fontaine, G., Villeneuve, B., Landstreet J.D., Taylor R.H. 1982, ApJS 49, 259

Harmanec, P. 1983, Hvar Obs. Bull. 7, 55

Harmanec, P. 1988, Bull. Astron. Inst. Czechosl. 39, 329

Harmanec, P. 1994, in NATO ARW: The impact of long-term monitoring on variable stars research, ed. by C. Sterken \& M. de Groot, Kluwer Acad. Press, Dordrecht, p. 55

Harmanec, P., Horn, J., Koubský, P, Božić, H. 1986, IBVS No. 2912

Harmanec, P., Pavlovski, K., Božić, H., et al. 1997, JAD 3, 5

Hubert, A.M., Floquet, M. 1998, A\&A 335, 567

Hubert-Delplace, A.M., Hubert, H. 1979, An atlas of Be stars, Paris-Meudon Observatory

Koubský, P., Ballereau, D., Chauville, J., Horn, J. 1995, in Astrophysical Applications of Stellar Pulsation, IAU Coll. 155, ed. by R.S. Stobbie and P.A. Whitelock, ASP Conf. Ser. 83, 307

Percy, J.R., Coffin, B.L., Druckier, G.A., et al. 1988, PASP 100, 1555

Percy, J.R., Harlow, J., Hayhoe, K.A.S., et al. 1997, PASP 109, 1215

Pavlovski, K., Harmanec, P., Božić, H., et al. 1997, A\&AS 125, 75

Plaskett, J.S., Pearce, J.A. 1931, Publ. Dominion. Astrophys. Obs. 5, 1

Slettebak, A. 1982 ApJS 50, 55

Stellingwerf, R.F. 1978, ApJ 224, 953 